\title{
Agent-Based Modelling and Simulation to Assess the Impact of Parking Reservation System
}

\author{
Xun-You Ni ${ }^{1,2}$ and Daniel (Jian) Sun ${ }^{1,2,3}$ \\ ${ }^{1}$ State Key Laboratory of Ocean Engineering, School of Naval Architecture, Ocean \& Civil Engineering, Shanghai Jiao Tong University, \\ Shanghai 200240, China \\ ${ }^{2}$ China Institute of Urban Governance, Shanghai Jiao Tong University, Shanghai 200240, China \\ ${ }^{3}$ Center for ITS and UAV Applications Research, School of Naval Architecture, Ocean \& Civil Engineering, \\ Shanghai Jiao Tong University, Shanghai 200240, China \\ Correspondence should be addressed to Daniel (Jian) Sun; danielsun@sjtu.edu.cn
}

Received 16 March 2017; Revised 17 May 2017; Accepted 31 May 2017; Published 5 July 2017

Academic Editor: Zhi-Chun Li

Copyright (C) 2017 Xun-You Ni and Daniel (Jian) Sun. This is an open access article distributed under the Creative Commons Attribution License, which permits unrestricted use, distribution, and reproduction in any medium, provided the original work is properly cited.

\begin{abstract}
With the increasing popularity of smart phones, Parking Reservation System (PRS) becomes practical to reduce the travel time in cruising for vacant spaces. The aim of this study is to assess the impact of PRS explicitly. This paper was started with analyzing the processes of cruising for vacant spaces and making parking reservation decisions. The vehicles were divided into two categories: the intelligent vehicles and the regular ones. Only the intelligent vehicles have the ability to make a parking reservation beforehand, while the regular ones have to cruise for vacant spaces. All involved components were treated as different agents, including vehicles, parking lots, network, and management center. Based on this, agent-based simulation was introduced to evaluate the performances of the scenarios with different penetration rates. The simulation results indicate the average travel time increases with the improvement of the penetration rates for the regular vehicles. The assessment method presented in this study would assist in promoting the performances of PRS in urban areas.
\end{abstract}

\section{Introduction}

The shortage of parking spaces is one of the major ignored transportation issues in urban areas $[1,2]$, mainly because the situation gained less attention than the roadway planning at early periods [3]. Taking facility capacities and competition behavior into account $[4,5]$, drivers may find no vacant spaces when arriving at the target parking lot. Parking Guidance and Information System (PGIS) is a useful tool to assist drivers to identify the parking lot with vacant spaces $[6,7]$. Based on the commonly used display terminal Variable Message Sign (VMS), PGIS releases the same parking information to all drivers that passed by [8], which generally induces many vehicles to compete for the limited spaces. When the last vacant space in the target parking lot was occupied, the rest of the drivers have to divert to the alternative ones. In recent years, with the rapid development of intelligent in-vehicle terminals (e.g., smart phones), drivers can receive real-time information and even reserve a parking space in advance [9]. With the real-time information, traffic congestions and parking problems could be both alleviated $[10,11]$. Parking Reservation System (PRS) enables drivers to reserve a parking space beforehand. As a result, drivers can directly head to the reserved parking lot [12] and the travel time in cruising for vacant spaces decreases accordingly [13].

To investigate the responding strategies of the reservation requests, Teodorović and Lučić [14] proposed an inventory control system to maximize the parking revenue. An integer programming approach was adopted to determine the optimal strategies under different vehicle arrival patterns. By introducing a parking space management center to handle all reservation requests, a model was proposed to minimize the total user costs [15]. The cost function was formulated as a weight sum of walking distance and monetary cost for using parking spaces. The reservation rule is that drivers who are satisfied with the assignment results can reserve the 
parking space; otherwise, they have to wait until the next allocation point. Based on the analysis of the interactions among the parking lot, the central server, and the vehicular drivers, an algorithm of appropriately assigning the parking spaces was proposed [16]. The results indicate the parking lots were utilized efficiently and the traffic congestions were alleviated. Unfortunately, the existing research mainly focused on replying to the reservation requests, seldom presenting the simulation steps explicitly. Consequently, it is critical to investigate the reservation process and provide a practical analysis tool.

Agent-based simulation is suitable to model the activities of participants in Intelligent Transportation Systems (ITS) [17] and describe drivers' responses to the parking information [18]. With the real-time information, agent-based simulation was used to replicate drivers' behavior [19], in which drivers' preferences and the impact of the information influencing the route selection were reflected. To describe the behavior of each driver within a spatial environment, Benenson et al. [20] implemented the agent-based simulation as an ArcGIS application. The application can explicitly simulate the entire parking process, including entering into parking zone, driving towards the destination, cruising for vacant spaces, and departing from the network. Moreover, based on the agent-based simulation package MATsim, cruising for vacant spaces according to the parking lot properties was discussed [21]. As for the parking reservation, Sana et al. [22] defined five types of agents: vehicle, traffic, global traffic, parking, and station, and the last one takes charging of preventing the reserved spaces from being illegally occupied. However, the simulation was also not illustrated explicitly. This paper tries to assess the impact of PRS by introducing agent-based simulation to describe the drivers' responses to the parking information, as well as the processes of cruising for vacant spaces and making parking reservation decisions dynamically.

The remainder of this paper is structured as follows. Section 2 analyzes the processes of cruising for vacant spaces and making parking reservation decisions. In Section 3, all involved components were treated as different agents, including vehicles, parking lots, network, and management center. Agent-based simulation was introduced to describe the processes of cruising for vacant spaces and making parking reservation decisions. Section 4 takes Xujiahui CBD, Shanghai, China, as a numerical example to illustrate the proposed assessment algorithm. Finally, conclusions and recommendations for future work are provided in Section 5.

\section{Processes of Cruising for Vacant Spaces and Making Parking Reservation Decisions}

Parking choice behavior is one of the hot issues for parking research, and the related theories and methods considering the real-time information are constantly enriched and perfected [23]. The rules of choosing parking lots are varied, as drivers prefer to choose the parking lot with vacant spaces [24] while the parking lot status changes with time, and consequently drivers often have to cruise for vacant spaces [25]. With the assistance of PRS, the vehicles configured with

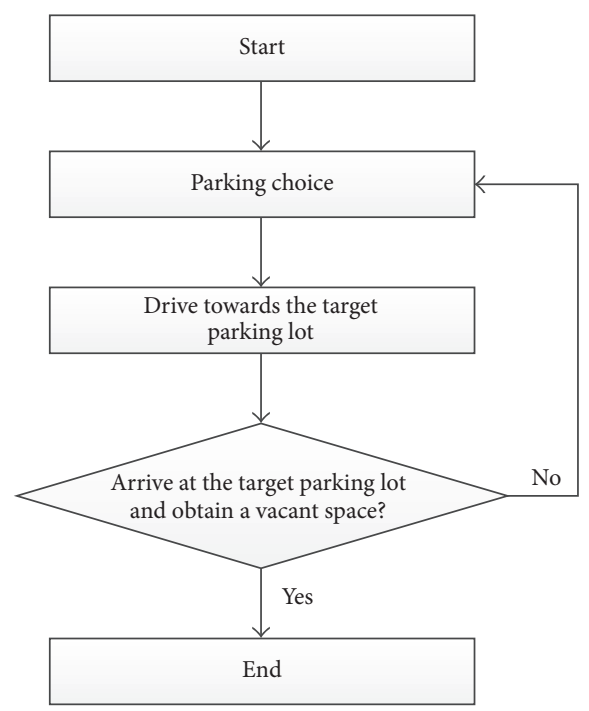

FIGURE 1: Process of cruising for vacant spaces.

smart phones can send a reservation request and probably avoid the cruising process. The vehicles were divided into two categories: the intelligent vehicles and the regular ones. Only the intelligent vehicles have the ability to make a parking reservation decision, while the regular ones have to cruise for vacant spaces. Processes of cruising for vacant spaces and making parking reservation decisions are analyzed in Sections 2.1 and 2.2.

2.1. Cruising for Vacant Spaces. Without the assistance of PRS, the cruising process is presented in Figure 1.

Figure 1 presents the process of cruising for vacant spaces. In general, drivers who enter into the parking zone should make a parking choice decision. The rule of making parking choice decisions is based on whether the target parking lot is still desirable. If the target parking lot is desirable, drivers insist on selecting the target parking lot; otherwise, a parking choice is reconducted to minimize the parking disutility. By integrating a perceived waiting time, the parking disutility was calculated as follows [26]:

$$
\mu_{i j k}=a_{1} t_{i j}+a_{2} d_{j}+a_{3} \frac{f_{j}}{T}+a_{4} w_{j k},
$$

where $\mu_{i j k}$ is the parking disutility travelling from intersection $i$, selecting parking lot $j$, with destination $k, t_{i j}$ is the travel time from intersection $i$ to parking lot $j, d_{j}$ is the perceived waiting time at parking lot $j, f_{j}$ is the fee charged at parking lot $j, T$ is the value of time, $w_{j k}$ is the walking time from parking lot $j$ to destination $k$, and $a_{1}, a_{2}, a_{3}$, and $a_{4}$ denote the relative important weighting coefficients of travel time, waiting time, fee charged, and walking time.

Moreover, the perceived waiting time was calculated as follows:

$$
d_{j}= \begin{cases}D, & \text { if parking lot status is full } \\ 0, & \text { otherwise }\end{cases}
$$


where $D$ denotes the perceived waiting time at the parking lot without vacant spaces.

For each vehicle, the comparison between the alternative parking lot and the target one is carried out to assist the parking choice decision-making. The alternative parking lot superior to the target one is expressed as

$$
\mu_{i j k}<\mu_{i j^{*} k} \text {, }
$$

where $\mu_{i j^{*} k}$ is the parking disutility travelling from intersection $i$, insisting on the target parking lot $j^{*}$, with destination $k$.

To describe driving towards the target parking lot, the spatial position is updated on the network. The updated algorithm is set as follows: if the vehicle arrives at an intersection, select the shortest route leading to the target parking lot and access the next link on the route. The shortest route is selected according to the Dijkstra algorithm [27]. If the vehicle is on a road link, move forwards according to the link average speed. The vehicular position is defined as the distance travelling from the upstream intersection. Moreover, if the distance is larger than the link length, the vehicular position is set equal to 0 , which indicates the vehicle has reached the downstream intersection. If the vehicle arrives at the target parking lot and acquires a vacant space, the cruising process ends; otherwise, the driver has to make another parking choice decision and continue cruising for vacant spaces.

2.2. Making Parking Reservation Decisions. For the intelligent vehicles, the reservation process is comparably simplified, as shown in Figure 2.

Figure 2 presents the process of making parking reservation decisions. Drivers who enter into the parking zone should make a parking choice decision according to the rule mentioned and send a reservation request. The superior rule is further defined as the alternative parking lot with smaller parking disutility except for the following condition: the target parking lot was reserved but the alternative one has no vacant spaces. If the target parking lot becomes undesirable, drivers make a parking choice decision to minimize the parking disutility and send a new reservation request; otherwise, they insist on selecting the target parking lot. The responding rule of the reservation requests is defined as follows: if the status of the target parking lot is available, the request is accepted; otherwise, it is rejected [28]. Then, they drive towards the target parking lot according to the updated algorithm. If drivers arrive at the target parking lot, they would directly park their vehicles; otherwise, the reservation process continues.

\section{Agent-Based Simulation Model}

Since drivers prefer to select the parking lot with vacant spaces and the parking lot status changes with time, it is essential to describe the processes of cruising for vacant spaces and making parking reservation decisions dynamically. Agent-based simulation modelled drivers' behavior by treating each vehicle as an autonomous agent, which is

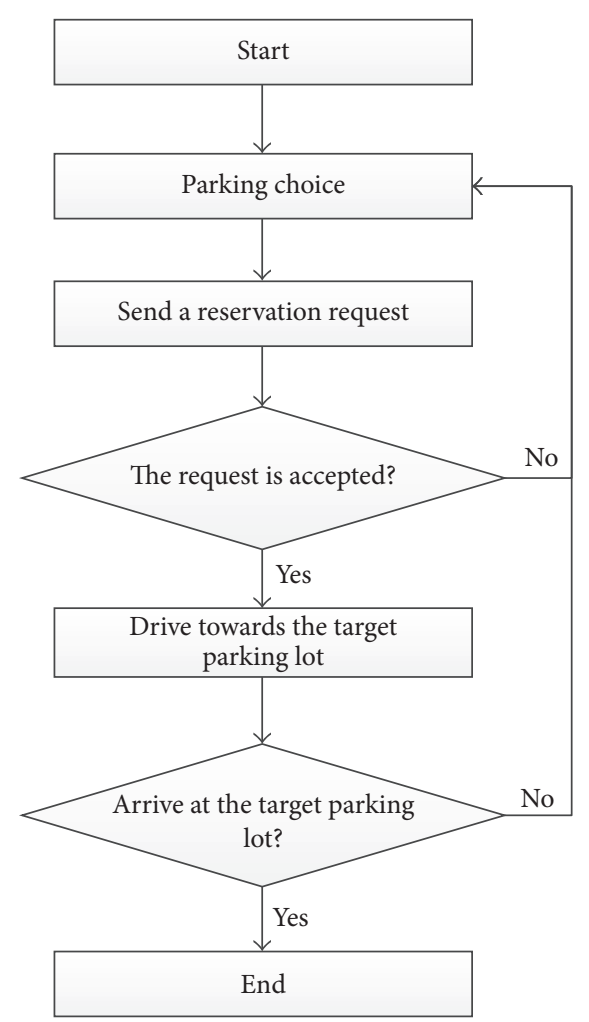

FIgURE 2: Process of making parking reservation decisions.

extensible according to the actual project demands. With the assistance of the agent-based simulation, this paper focuses on evaluating the performances of the scenarios with different penetration rates. Additionally, the penetration rate is the ratio of the intelligent vehicles that are able to make parking reservation decisions.

3.1. Agent Design. To implement the agent-based simulation, agents which describe the activities of participants have to be designed. For instance, the intelligent vehicles receive realtime information and send the reservation requests via smart phones, while the regular ones have to cruise for vacant spaces. The parking and traffic information mainly includes the parking lot status and the link average speed, while the remaining information is static. Management center takes charge of replying to the reservation requests. All the involved components were treated as different agents, including vehicles, parking lots, network, and management center, as follows.

3.1.1. Vehicle Agent. Each vehicle is treated as an autonomous agent with six attributes: origin, destination, an intelligent flag indicating whether the vehicle is an intelligent one, target parking lot, entering time, and departing time. As for the attributes, the first three are static while the rest are the dynamic ones. Moreover, the first three are known for each vehicle and the rest are updated according to the simulation conditions. The vehicle agents move forwards on the road network individually. When entering into the parking zone, the vehicular attributes are initialized. Then, the vehicles are 
loaded on the network and travel along the route leading to the target parking lot. If arriving at the downstream intersection, the vehicle would access the next link directly connected.

3.1.2. Parking Lot Agent. The parking lot agent also includes two types of attributes: the static and the dynamic ones. The static attributes include name, location, capacity, and fare charged for each parking lot, while the dynamic one is mainly the parking lot status. If no vacant spaces within the specified parking lot, the status becomes FULL; otherwise, the status is available.

3.1.3. Network Agent. Road network was treated as network agent, mainly consisting of eight attributes: link length, link lanes, link density, link free-flow speed, link minimal speed, link average speed, intersection position, and the connecting relationships between the links and the intersections. The link density and the average speed change with time, while the remaining attributes are static, and the link density is defined as the number of vehicles within one unit length of the link, which can be used to determine the link average speed.

3.1.4. Management Center Agent. Management center agent is responsible for handling the reservation requests and updating the reservation list. To reserve at least one space in the reserved parking lot and prevent the reserved spaces being illegally occupied, the rules of attaching/detaching operations are defined as follows: if the reservation requests were accepted, attach the vehicles to the reserved parking lot; otherwise, drivers make a parking choice decision according to the rule discussed. When the intelligent vehicles arrive at the reserved parking lot, they have to be detached from the parking lot. The attaching/detaching operations form the reservation list, which denotes the reservation relationships of the intelligent vehicles and the reserved parking lots at the simulation iterations. In the reservation list, the spaces are named as the reserved ones.

3.2. Agent-Based Simulation Framework. In the simulation environment, only vehicle agents move dynamically. The possible states of the vehicle agents are entering into the parking zone, cruising for vacant spaces or making parking reservation decisions, heading to the target parking lot, and departing from the network. By incorporating the agent design and the processes of cruising for vacant spaces and making parking reservation decisions, the agent-based simulation is illustrated in Figure 3. follows.

The overall process of the simulation is summarized as

Step 0 (initialization). Set the link flow, the link density, the total travel time, and the total walking time equal to 0 . Then, initialize the current iteration to 1 second, the link travel time to the free-flow travel time, and the number of vacant spaces in the parking lots to the capacities. Additionally, the interval iteration is set as 1 second.
Step 1. Update the parking lot status and traffic conditions. Parking lot agent, management center agent, and network agent take charge of updating the parking lot status, the reservation list, and the traffic conditions.

(a) Update the parking lot status. The number of vacant spaces is computed as follows:

$$
q_{j}=\max \left(q_{j}^{*}-\rho_{j}-h_{j}+\omega_{j}, 0\right),
$$

where $\rho_{j}$ is the number of the reserved spaces in parking lot $j, h_{j}$ is the number of vehicles arriving at parking lot $j, \omega_{j}$ is the number of vehicles departing from parking lot $j$, and $q_{j}$ and $q_{j}^{*}$ are the number of vacant spaces in parking lot $j$ at current and previous iterations, respectively.

The parking lot status is updated according to the number of vacant spaces. If there are no vacant spaces in the parking lot, the status becomes FULL; otherwise, the status is available.

(b) Update the traffic conditions. The link density is calculated as follows:

$$
g_{a}=\frac{x_{a}}{\eta_{a} L_{a}},
$$

where $g_{a}$ is the vehicular density on link $a, x_{a}$ is the traffic flow on link $a, \eta_{a}$ is the number of lanes of link $a$, and $L_{a}$ is the length of link $a$.

To capture the link average speed varying with the density, Li et al. [29] proposed a traffic flow model as follows:

$$
\begin{aligned}
& v_{a} \\
& = \begin{cases}v_{a f}, & g_{a} \leq g_{a b} \\
v_{a 0}+\left(v_{a f}-v_{a 0}\right)\left(1-\frac{g_{a}-g_{a b}}{g_{a j}-g_{a b}}\right)^{\gamma}, & g_{a b}<g_{a} \leq g_{a j},\end{cases}
\end{aligned}
$$

where $v_{a}$ is the average speed on link $a, v_{a 0}$ is the minimal speed on link $a, v_{a f}$ is the free-flow speed on link $a, g_{a b}$ is the density breakpoint on link $a, g_{a j}$ is the jam density on link $a$, and $\gamma$ is the parameter.

(c) Update the reservation list according to the attaching/detaching operations (as discussed in Section 3.1).

Step 2. Vehicles enter into the parking zone. The origin, the destination, and the intelligent flag are known for each vehicle. If the vehicle is an intelligent one, initialize the intelligent flag equal to 1 ; otherwise, the value is 0 . Then, load the vehicles on the network and set the attribute entering time equal to the current time.

Step 3. Examine whether the vehicles arrive at the target parking lot. For each intelligent vehicle, if drivers arrive at the reserved parking lot, their vehicles would be directly parked and detached from the parking lot. For each regular one, if drivers obtain a vacant space, the cruising process ends. 


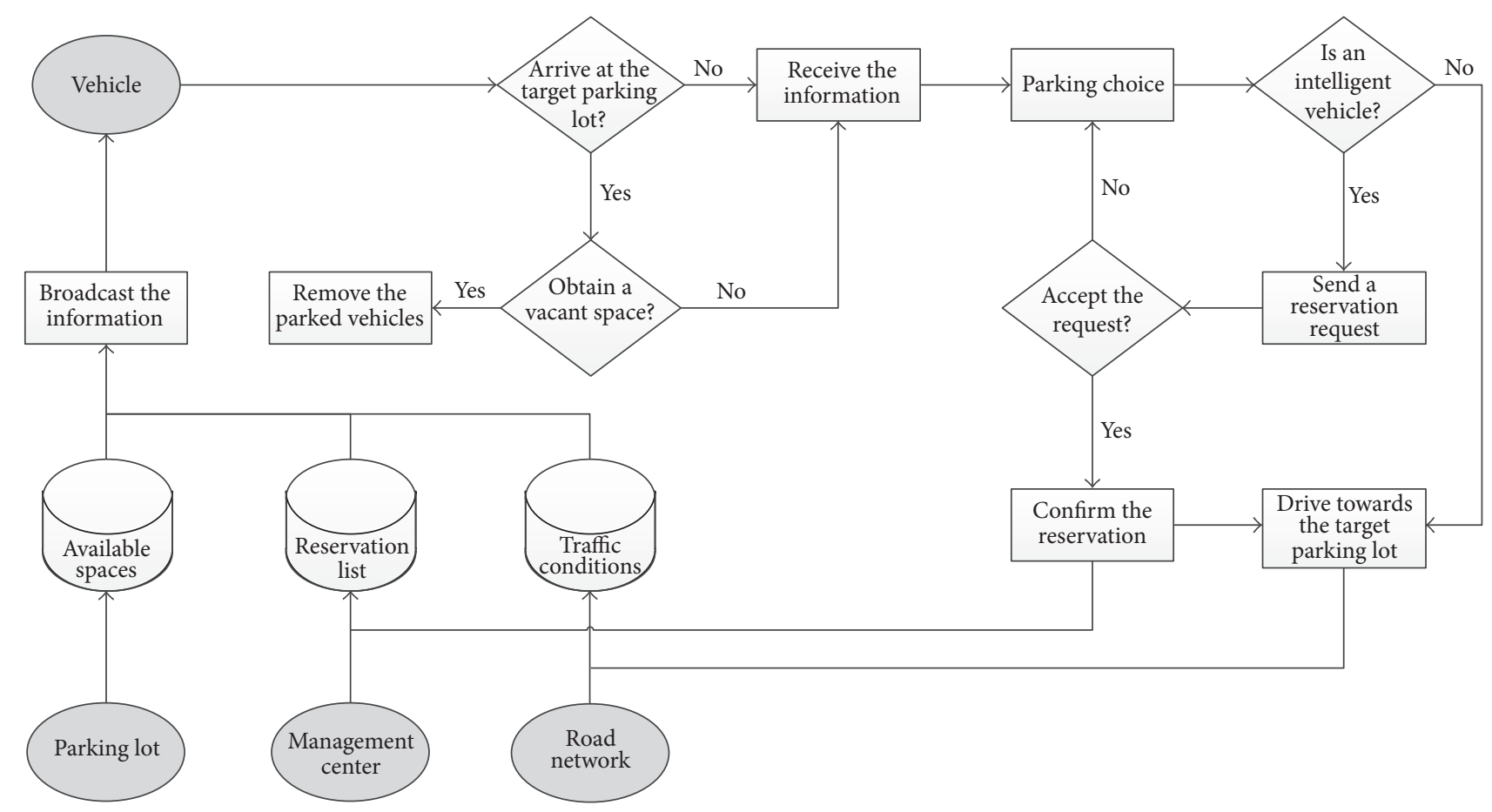

FIGURE 3: Framework of the agent-based simulation.

Then, the departing time is set equal to the current time and the parked vehicles are removed from the network. The total travel time is calculated as follows:

$$
T=T^{*}+T_{2}-T_{1},
$$

where $T$ and $T^{*}$ are the total travel time at the current and previous iterations and $T_{1}$ and $T_{2}$ are the entering time and departing time for the vehicle, respectively.

The total walking distance is computed as follows:

$$
W=W^{*}+\Gamma_{j k},
$$

where $\Gamma_{j k}$ is the walking distance from the target parking lot $j$ to destination $k$ for the vehicle and $W$ and $W^{*}$ are the total walking distance at current and previous iterations, respectively.

Step 4. Make parking choice decisions according to the rule discussed in Sections 2.1 and 2.2. Additionally, if an alternative parking lot is reserved, the vehicle should be detached from the target parking lot and attached to the alternative one.

Step 5. Drive towards the target parking lot according to the update algorithm, as presented in Section 2.1.

Step 6 (stop criteria). If all the parking lots are unavailable, stop and export the performances; otherwise, continue the simulation and go to Step 1. The performances are indicated by two indicators: average travel time and average walking distance. Travel time is one of the major indicators to assess the traffic conditions in the congested urban areas, and walking distance determines the reasonability of the parking guidance scenarios. For the parked vehicles, the average travel time is the total travel time divided by the number of vehicles. Similarly, the average travel distance is computed.

\section{Numerical Example}

Xujiahui CBD, Shanghai, China, was built in the 1990s, providing the comprehensive commercial services, such as shopping, entertainment, and business. The core area starts from Yishan Rd in the west and ends at Wanping Rd and starts from Guangyuan Rd in the north and ends at Lingling Rd. It covers 4.04 square kilometers, with the radius is around 1000 meters, and the center locates at an intersection intersected by five roads: Huashan Rd, Hongqiao Rd, North Caoxi Rd, Zhaojiabang Rd, and Hengshan Rd. Location and layout of Xujiahui CBD are presented in Figure 4.

Figure 5 presents the network of Xujiahui CBD, consisting of 101 nodes, 159 road links, 5 origins (from 1 to 5), 2 destinations (from 6 to 7), and 6 major parking lots (from 8 to 13), with 2768 parking spaces in total. Labels along the links are the lengths, with unit meter. Parking demands have 10 Origin-Destination (OD) pairs. For simplification, the arrival rate for each OD pair was assumed as 2 vehicles per minute and the departing ratio for each parking lot was set as 0.5 vehicles per minute. The bold lines denote the arterials, and the ordinary lines are the branches. Capacities and fees charged for the parking lots were obtained and are presented in Table 1.

As for the penetration rates, five typical scenarios were evaluated as $0.2,0.4,0.6,0.8$, and 1.0. The scenario with penetration rate 0.2 was named as Scenario 1, and the 


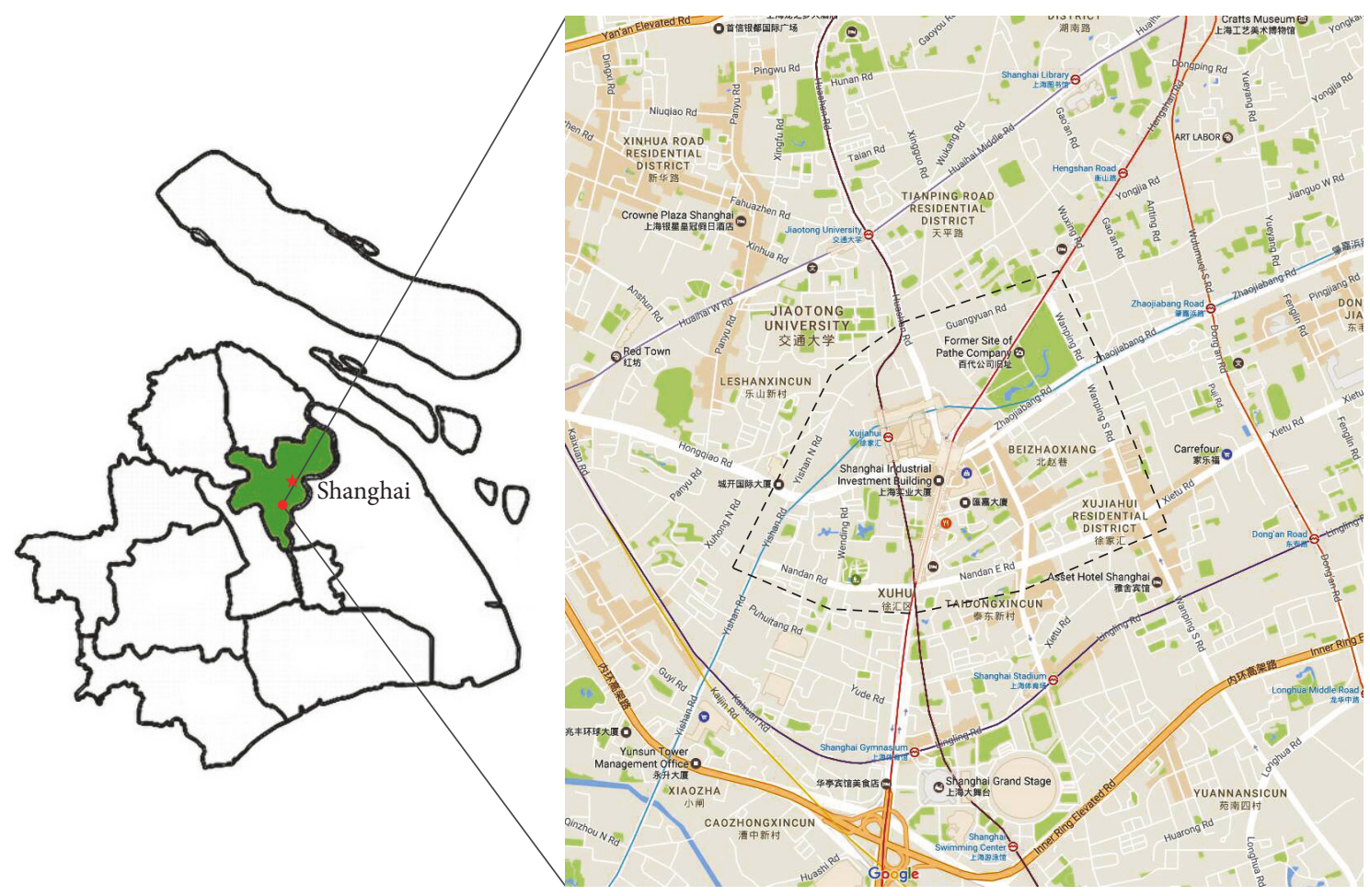

* Municipal government
- Location
- - Boundary of the core area

Figure 4: Location and layout of Xujiahui CBD, Shanghai, China.

TABLE 1: Capacities and fees charged for the parking lots.

\begin{tabular}{lcc}
\hline Parking lot index & Capacity & Fee $(\mathrm{RMB} / \mathrm{h})$ \\
\hline 8 & 1260 & 10 \\
9 & 500 & 9 \\
10 & 191 & 15 \\
11 & 333 & 10 \\
12 & 250 & 15 \\
13 & 234 & 10 \\
\hline
\end{tabular}

remaining scenarios were denoted as Scenario 2, Scenario 3, Scenario 4, and Scenario 5 in sequence. By implementing the agent-based simulation to evaluate the five scenarios, the number of the reserved spaces was obtained and is shown in Figure 6.

For all the scenarios, the number of the reserved spaces increases when the target parking lot has vacant spaces and decreases under the condition that the majority of the intelligent vehicles arrive at the target parking lot. Between the trends rise and decline, most intelligent vehicles are heading to the target parking lot with the status FULL. At the stage, the indicator reaches the maximum value. Moreover, the fluctuations indicate the intelligent vehicles are able to change the reservation decisions and reserve the alternative parking lot. It is obvious that P8 and P10 are the most popular parking lots, while the remaining parking lots are comparably less utilized.

The parking demands served by each parking lot are shown in Table 2.

The performances of PRS are presented in Figures 7 and 8 . Figure 7 presents the average travel time with the scenarios. For the regular vehicles, the average travel time increases with the improvement of the penetration rates. For the intelligent ones, the indicators are roughly equal. That is, too many intelligent vehicles reserve the desirable parking lots in advance, which does harm on the regular vehicles. Moreover, the average travel time for the regular vehicles is larger than the value for the intelligent vehicles, owing to the process of cruising for vacant spaces.

The average walking distances were obtained and are shown in Figure 8. With the increasing of the penetration rates, no large difference occurs in the average walking distance for the scenarios. For the intelligent vehicles and the regular ones, the maximum growth values of the indicator are 44.68 meters and 20.10 meters, respectively. Consequently, it may be acceptable for drivers in the congested urban areas.

\section{Conclusions}

With the wide ownership and usage of smart phones, PRS becomes practical to reduce the travel time in cruising for vacant spaces. This paper assesses the impact of PRS by 


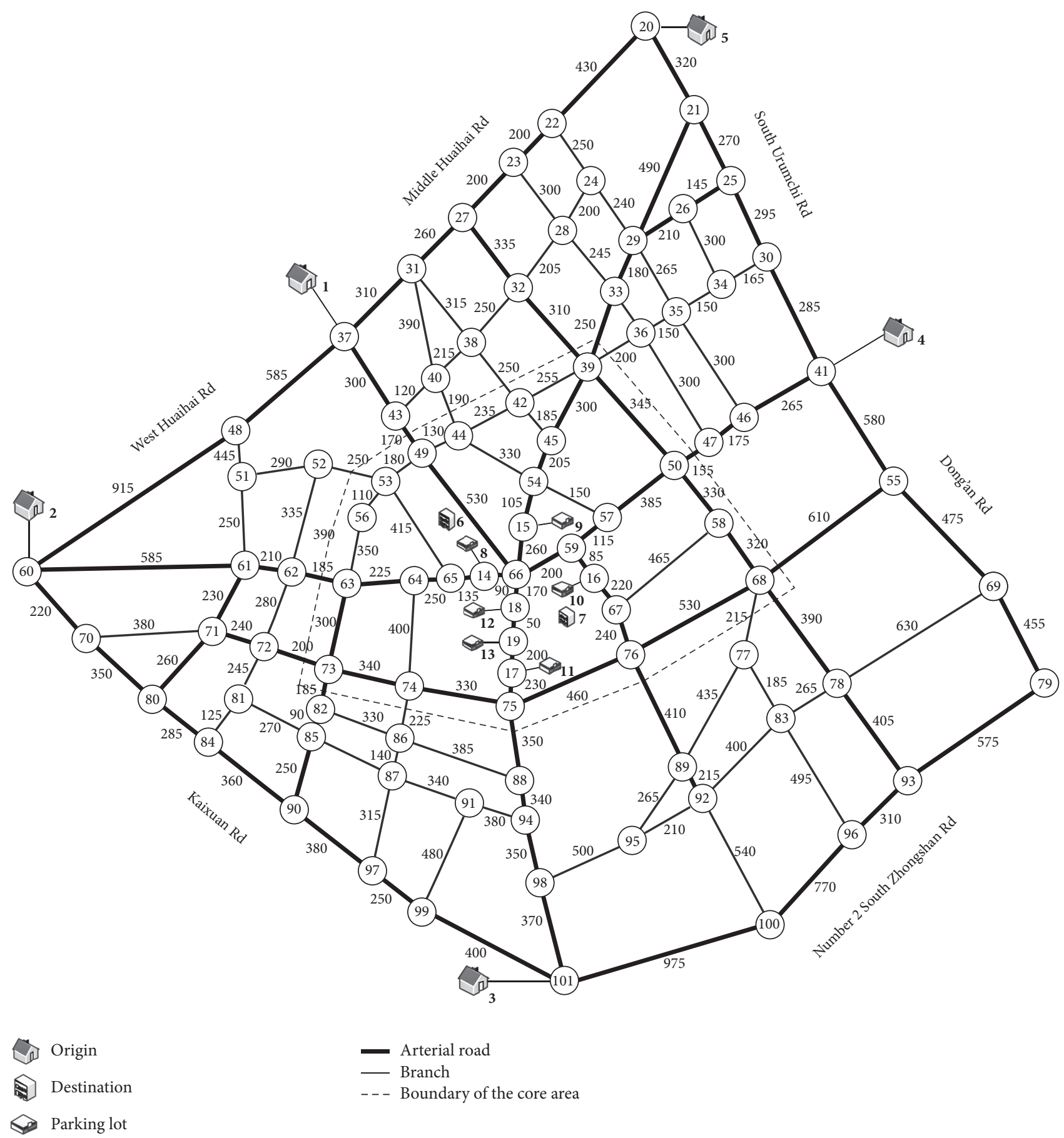

FIgure 5: Network of Xujiahui CBD, Shanghai, China.

introducing agent-based simulation to model the drivers' responses to the parking information, as well as the processes of cruising for vacant spaces and making parking reservation decisions dynamically. The involved functionality components were treated as different agents, including vehicles, parking lots, network, and management center. Conclusions are drawn as follows:

(i) To describe the vehicles moving on the network dynamically, the processes of cruising for vacant spaces and making parking reservation decisions were analyzed. Vehicles were divided into two categories: the intelligent vehicles and the regular ones. Only the intelligent vehicles have the ability to make a parking reservation decision, while the regular ones have to cruise for vacant spaces.

(ii) Agent-based simulation was introduced to describe the processes of cruising for vacant spaces and making reservation decisions dynamically. Of all 


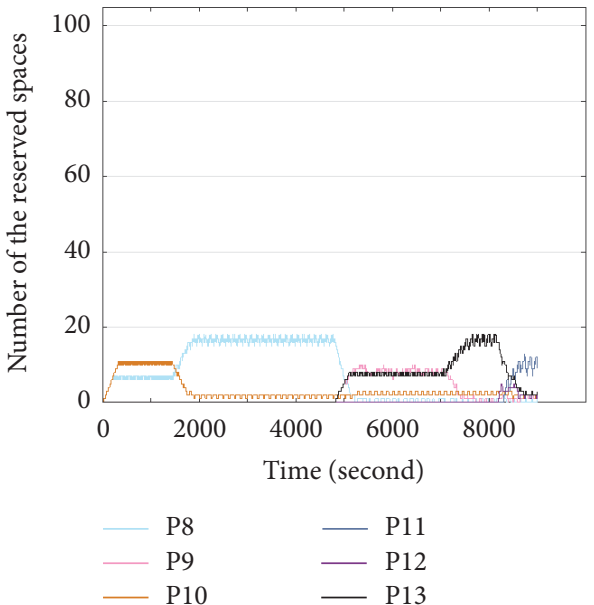

(a)

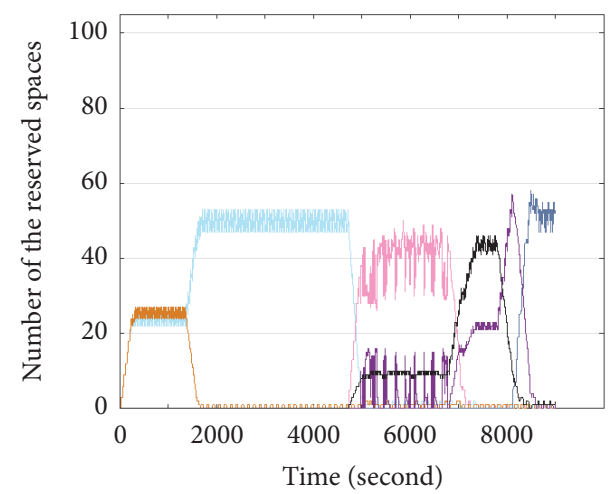

$\begin{aligned} \text { P8 } & - \text { P11 } \\ \text { P9 } & \text { P12 } \\ - \text { P10 } & - \text { P13 }\end{aligned}$

(c)
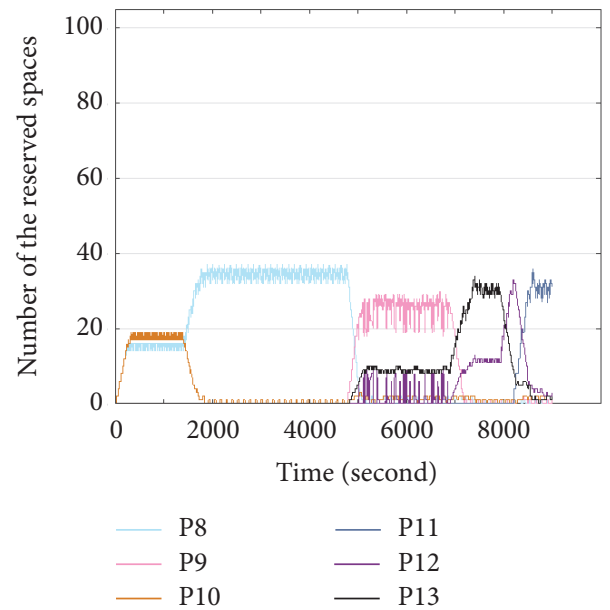

(b)
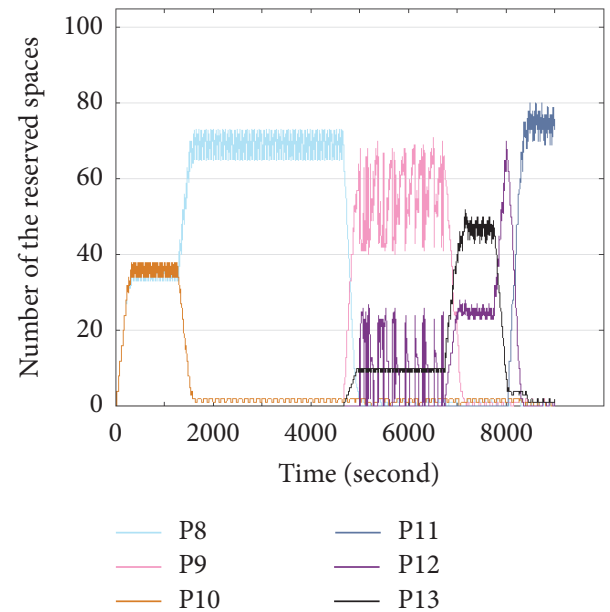

(d)

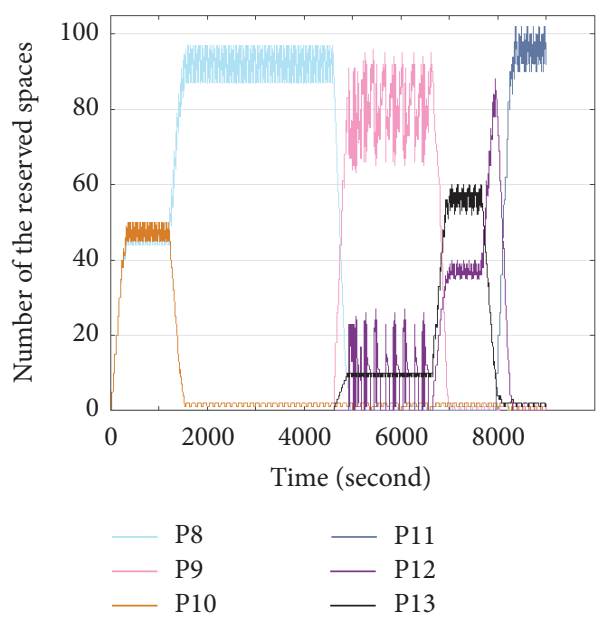

(e)

FiguRE 6: Number of the reserved spaces with the scenarios, (a) Scenario 1, (b) Scenario 2, (c) Scenario 3, (d) Scenario 4, and (e) Scenario 5. 
TABLE 2: Parking demands served by each parking lot.

\begin{tabular}{|c|c|c|c|c|c|c|c|}
\hline \multirow{2}{*}{ Scenario } & \multirow{2}{*}{ Vehicle type } & \multicolumn{6}{|c|}{ Parking lot index } \\
\hline & & 8 & 9 & 10 & 11 & 12 & 13 \\
\hline \multirow{2}{*}{1} & Intelligent & 278 & 71 & 109 & 9 & 7 & 107 \\
\hline & Regular & 1055 & 462 & 153 & 210 & 275 & 157 \\
\hline \multirow{2}{*}{2} & Intelligent & 548 & 184 & 154 & 43 & 81 & 150 \\
\hline & Regular & 785 & 350 & 108 & 174 & 201 & 115 \\
\hline \multirow{2}{*}{3} & Intelligent & 816 & 292 & 196 & 87 & 162 & 188 \\
\hline & Regular & 517 & 243 & 67 & 127 & 120 & 78 \\
\hline \multirow{2}{*}{4} & Intelligent & 1078 & 397 & 232 & 149 & 232 & 229 \\
\hline & Regular & 255 & 137 & 31 & 67 & 50 & 37 \\
\hline 5 & Intelligent & 1333 & 533 & 263 & 216 & 284 & 267 \\
\hline
\end{tabular}

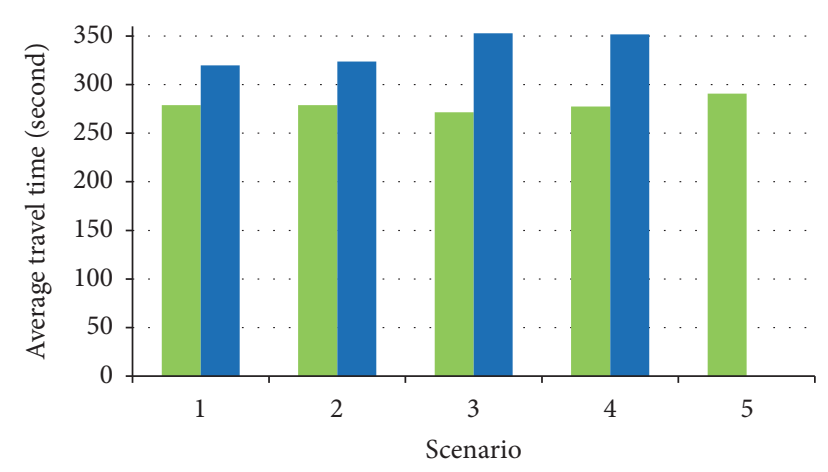

Intelligent vehicles

- Regular vehicles

FIgURE 7: Average travel time with the scenarios.

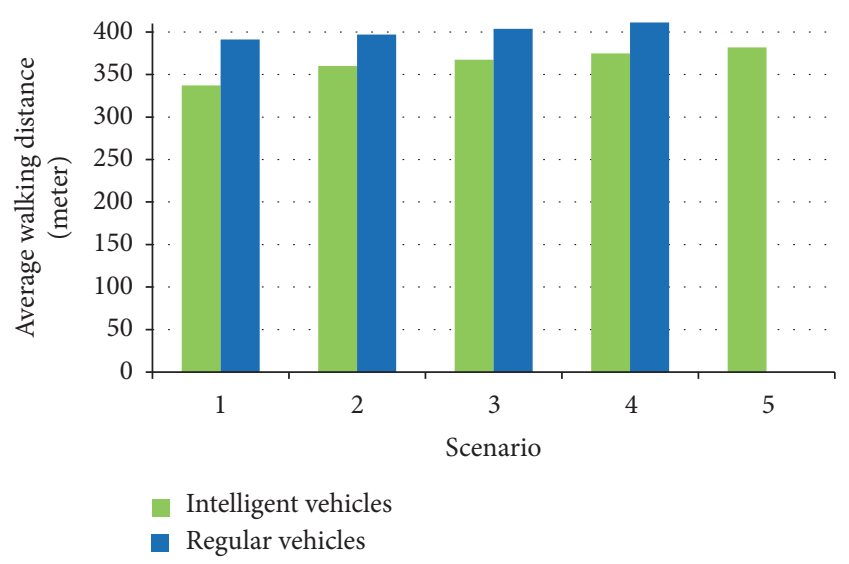

Figure 8: Average walking distance with the scenarios.

involved components, only the vehicle agents can move dynamically.

(iii) To reserve at least one space in the reserved parking lot, the rules of attaching/detaching were defined. If the reservation requests were accepted, the vehicles are attached to the reserved parking lot; otherwise, drivers make a parking choice decision according to the rule discussed. Moreover, if the intelligent vehicles arrive at the target parking lot, they have to be detached from the parking lot.

The simulation results indicate the average travel time increases with the improvement of the penetration rates for the regular vehicles. While the results are promising, further studies may be conducted to improve the performances of the proposed method. As a foundation of cruising for vacant spaces and making parking reservation decisions is the parking disutility function, it is critical to adjust the parameters to describe the processes accurately. Moreover, the parameters of the traffic flow model used in this study may be further investigated. With large amount of field data, agent-based simulation may be studied to evaluate the performances of PRS more practically. Further studies may implement the proposed algorithm in a real environment, and then the performances with more traffic data can be assessed.

\section{Disclosure}

Any opinions, findings, and conclusions or recommendations expressed in this paper are those of the authors and do not necessarily reflect the views of the sponsors.

\section{Conflicts of Interest}

The authors declare that there are no conflicts of interest regarding the publication of this paper.

\section{Acknowledgments}

The authors would like to express their appreciation to Professor Zhong-Ren Peng from Department of Urban and Regional Planning, University of Florida, for his valuable suggestions during the conduct of this study. Additionally, the support from the Humanities and Social Science Research Project, Ministry of Education, China (15YJCZH148), the Philosophy and Social Science Research Project of Shanghai, China (2014BGL009), and the Shanghai Municipal Natural Science Foundation (17ZR1445500) is greatly appreciated. 


\section{References}

[1] D. J. Sun, J.-M. Cheng, and Y. Zhang, "An AHP-Fuzzy comprehensive evaluation model for parking lots in urban CBD area," Advances in Transportation Studies, vol. 2015, no. 37, pp. 141-156, 2015.

[2] A. O. Kotb, Y.-C. Shen, X. Zhu, and Y. Huang, "IParker-A New Smart Car-Parking System Based on Dynamic Resource Allocation and Pricing," IEEE Transactions on Intelligent Transportation Systems, vol. 17, no. 9, pp. 2637-2647, 2016.

[3] Z. Qian and R. Rajagopal, "Optimal occupancy-driven parking pricing under demand uncertainties and traveler heterogeneity: A stochastic control approach," Transportation Research Part B: Methodological, vol. 67, pp. 144-165, 2014.

[4] F. Shi, G.-M. Xu, and H. Huang, "An augmented Lagrangian origin-based algorithm for link-capacitated traffic assignment problem," Journal of Advanced Transportation, vol. 49, no. 4, pp. 553-567, 2015.

[5] F. Leurent and H. Boujnah, "A user equilibrium, traffic assignment model of network route and parking lot choice, with search circuits and cruising flows," Transportation Research Part C: Emerging Technologies, vol. 47, no. 1, pp. 28-46, 2014.

[6] S. A. Bagloee, A. Ceder, and C. Bozic, "Effectiveness of en route traffic information in developing countries using conventional discrete choice and neural-network models," Journal of Advanced Transportation, vol. 48, no. 6, pp. 486-506, 2014.

[7] Z.-C. Li, H.-J. Huang, and W. H. K. Lam, "Modelling heterogeneous drivers' responses to route guidance and parking information systems in stochastic and time-dependent networks," Transportmetrica, vol. 8, no. 2, pp. 105-129, 2012.

[8] X.-Y. Ni, D. Sun, and Z.-R. Peng, "An improved incremental assignment model for parking variable message sign location problem," Journal of Advanced Transportation, vol. 49, no. 7, pp. 817-828, 2015.

[9] B. Zou, N. Kafle, O. Wolfson, and J. J. Lin, "A mechanism design based approach to solving parking slot assignment in the information era," Transportation Research Part B: Methodological, vol. 81, pp. 631-653, 2015.

[10] Z. Zhu, B. Peng, C. Xiong, and L. Zhang, "Short-term traffic flow prediction with linear conditional Gaussian Bayesian network," Journal of Advanced Transportation, vol. 50, no. 6, pp. 1111-1123, 2016.

[11] F. Caicedo, C. Blazquez, and P. Miranda, "Prediction of parking space availability in real time," Expert Systems with Applications, vol. 39, no. 8, pp. 7281-7290, 2012.

[12] A. Schlote, C. King, E. Crisostomi, and R. Shorten, "Delaytolerant stochastic algorithms for parking space assignment," IEEE Transactions on Intelligent Transportation Systems, vol. 15, no. 5, pp. 1922-1935, 2014.

[13] M. Gallo, L. D’Acierno, and B. Montella, "A multilayer model to simulate cruising for parking in urban areas," Transport Policy, vol. 18, no. 5, pp. 735-744, 2011.

[14] D. Teodorović and P. Lučić, "Intelligent parking systems," European Journal of Operational Research, vol. 175, no. 3, pp. 1666-1681, 2006.

[15] Y. Geng and C. G. Cassandras, "New 'smart parking' system based on resource allocation and reservations," IEEE Transactions on Intelligent Transportation Systems, vol. 14, no. 3, pp. 1129-1139, 2013.

[16] J.-H. Shin and H.-B. Jun, "A study on smart parking guidance algorithm," Transportation Research Part C: Emerging Technologies, vol. 44, pp. 299-317, 2014.
[17] Y. Yu, A. El Kamel, G. Gong, and F. Li, "Multi-agent based modeling and simulation of microscopic traffic in virtual reality system," Simulation Modelling Practice and Theory, vol. 45, pp. 62-79, 2014.

[18] D. J. Sun, X.-Y. Ni, and L.-H. Zhang, "A discriminated release strategy for parking variable message sign display problem using agent-based simulation," IEEE Transactions on Intelligent Transportation Systems, vol. 17, no. 1, pp. 38-47, 2016.

[19] H. Dia, "An agent-based approach to modelling driver route choice behaviour under the influence of real-time information," Transportation Research C: Emerging Technologies, vol. 10, no. 56, pp. 331-349, 2002.

[20] I. Benenson, K. Martens, and S. Birfir, "PARKAGENT: An agent-based model of parking in the city," Computers, Environment and Urban Systems, vol. 32, no. 6, pp. 431-439, 2008.

[21] R. Waraich and K. Axhausen, "Agent-based parking choice model," Transportation Research Record, no. 2319, pp. 39-46, 2012.

[22] B. Sana, H. Riadh, and M. Rafaa, "Intelligent parking management system by multi-agent approach: the case of urban area of Tunis," in Proceedings of the 2014 International Conference on Advanced Logistics and Transport (ICALT '14), pp. 65-71, Hammamet, Tunisia, May 2014.

[23] M. Ottomanelli, M. Dell'Orco, and D. Sassanelli, "Modelling parking choice behaviour using possibility theory," Transportation Planning and Technology, vol. 34, no. 7, pp. 647-667, 2011.

[24] S. Tang, T. Rambha, R. Hatridge, S. D. Boyles, and A. Unnikrishnan, "Modeling parking search on a network by using stochastic shortest paths with history dependence," Transportation Research Record, vol. 2467, pp. 73-79, 2014.

[25] R. Arnott and E. Inci, "An integrated model of downtown parking and traffic congestion," Journal of Urban Economics, vol. 60, no. 3, pp. 418-442, 2006.

[26] R. G. Thompson, K. Takada, and S. Kobayakawa, "Optimisation of parking guidance and information systems display configurations," Transportation Research Part C: Emerging Technologies, vol. 9, no. 1, pp. 69-85, 2001.

[27] Y. Sheffi, Urban Transportation Networks: Equilibrium Analysis with Mathematical Programming Methods, Prentice-Hall, Inc, Englewood Cliffs, NJ, USA, 1985.

[28] M. Kaspi, T. Raviv, and M. Tzur, "Parking reservation policies in one-way vehicle sharing systems," Transportation Research Part B: Methodological, vol. 62, pp. 35-50, 2014.

[29] A. C. Y. Li, L. Nozick, R. Davidson, N. Brown, D. A. Jones, and B. Wolshon, "Approximate solution procedure for dynamic traffic assignment," Journal of Transportation Engineering, vol. 139, no. 8, pp. 822-832, 2013. 


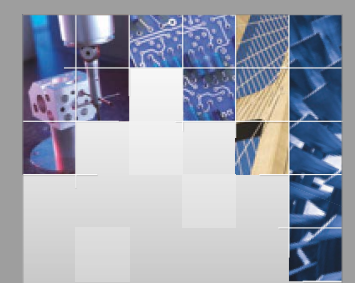

\section{Enfincering}
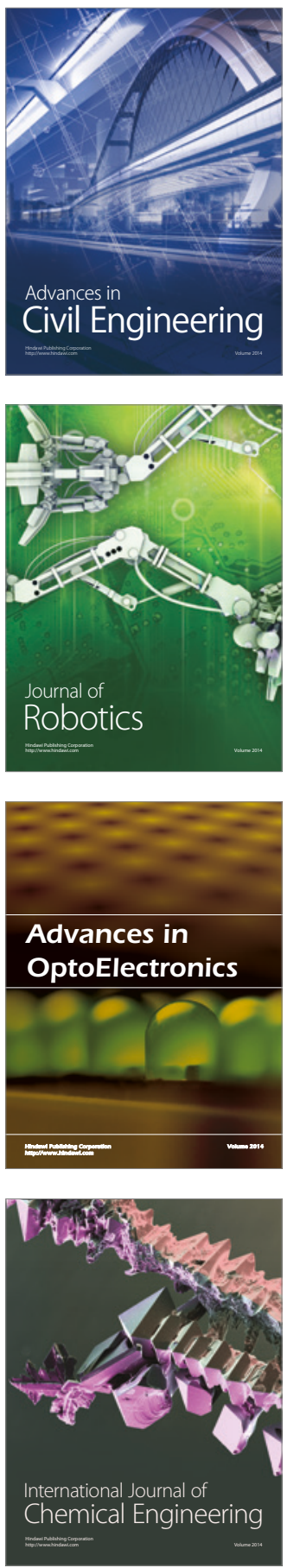

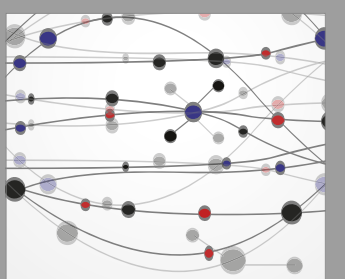

The Scientific World Journal

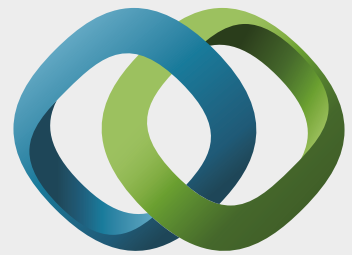

\section{Hindawi}

Submit your manuscripts at

https://www.hindawi.com
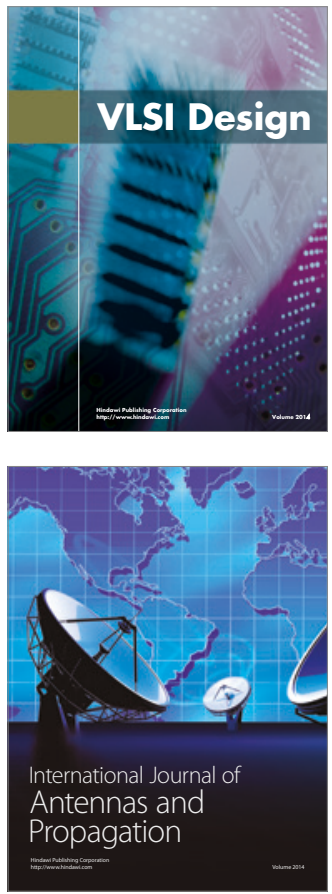

\section{Rotating}

Machinery
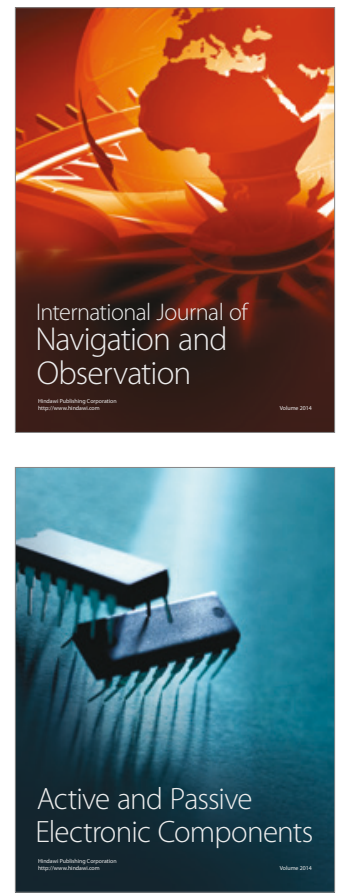
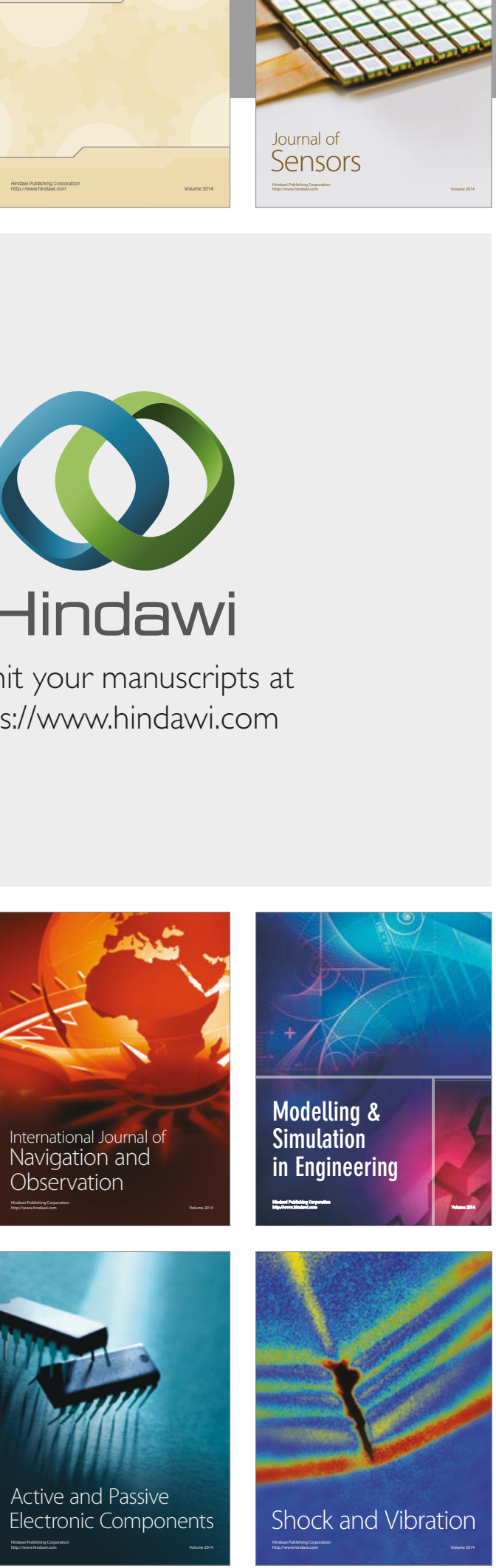
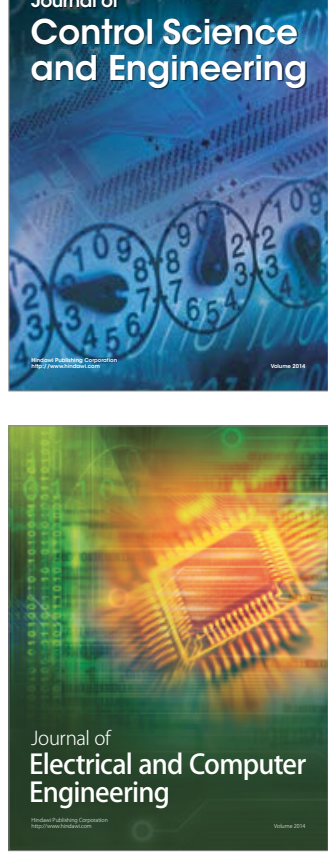

Distributed

Journal of

Control Science

and Engineering
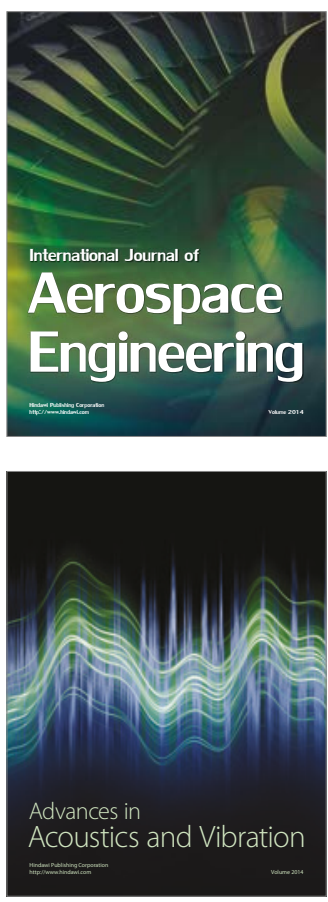

Sensor Networks 